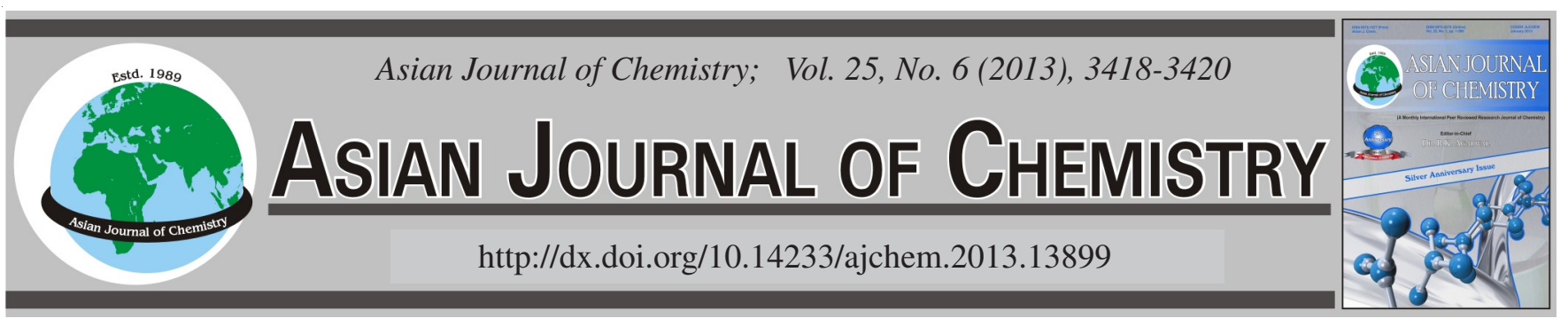

\title{
Optimization Solid Phase Extraction of Copper(II) Using Modified Nanoporous Silica Prior to Determination by Flame Atomic Absorption Spectrometry by Taguchi Technique
}

\author{
M.R. Sohrabi ${ }^{*}$, H. PASDAR, S.H. RoudBaraki and S. GHAJAR
}

Department of Chemistry, Islamic Azad University, North Tehran Branch, P.O. Box 1913674711, Tehran, Iran

*Corresponding author: Fax: +98 21 22222512; Tel: +98 21 22222151; E-mail: m.r.sohrabi2020@gmail.com

This paper deals with solid phase extraction coupled with atomic absorption spectrometry for determination of $\mathrm{Cu}$ (II) in trace level in aqueous solution. Design of experiment, based on Taguchi technique, is used to evaluate optimum conditions of separation. Design of experiment approach is a method to reduce the time and onus of experiments in comparison with one factor at a time optimization. For experimental parameters $\mathrm{pH}$, flow rate, time and absorbent dosage were applied at three levels to achieve suitable conditions of separation of $\mathrm{Cu}(\mathrm{II})$. Results indicate that flow rate parameter, at low level and time parameter, at high level, are more effective than other parameters at the best separation of $\mathrm{Cu}(\mathrm{II})$ which is equal to $96.3 \%$.

Key Words: Taguchi experimental design, Solid phase extraction, Nanoporous silica, Flame atomic absorption.

\section{INTRODUCTION}

In general, heavy metal ions are toxic, non-biodegradable and tend to be accumulated in the human vital organs, where they can act progressively over a long period through food chains. The determination of trace heavy metal ions in environmental of samples has received increasing attention ${ }^{1-3}$. However, due to the complexity of sample matrix and the frequently low concentrations of metals, there is a crucial need for the extraction and preconcentration of trace elements from matrix before their analysis using atomic absorption spectrometry or other techniques.

To obviate these problems, an effective extraction and preconcentration method is necessary. In the last two decades the solid phase extraction approach has gained rapid acceptance. It is considered an alternative to the classical liquid extraction technique, owing to several major advantages in application such as fast, simple, amenability to automation, low disposal costs and extraction time for sample preparation $^{4,5}$.

Operating parameters have significant influence on the separation. To achieve an effective performance, optimization of operating parameters is necessary. To establish better conditions by relating all the factors considered, numerous experiments have to be carried out with all the possible parameter combinations, which is not practical. Conventional experimental procedures, involve altering one factor at a time keeping all other factors constant, resulting in assessing the impact of those particular factors, which are time consuming and require more experimental sets. Design of experiments (DOE) is one approach, which helps to gain information about the optimized levels, by taking large number of variable ${ }^{6}$.

For procedures which are involving a limited number of variables at few levels, the factorial strategy is commonly used. This sequential approach tests every level of every variable (referred to as factors) against every level of every other factor. This approach increases the likelihood of finding the exact optima for each factor and is powerful at discovering interactions between factors. However, complex optimizations, involving four or more factors, each at three or more levels, require an increasing number of experiments using this approach. An alternative approach uses orthogonal arrays to reduce the number of experiments required, whilst avoiding a reduction in power that comes with using partial or fractional designs.

A commonly used orthogonal method is the Taguchi design ${ }^{7}$. Extensively used for engineering process optimization, it incorporates one primary experiment to study the main effects of each factor and to model some of the important interactions. Secondary Taguchi arrays can be designed from the primary results, to narrow the optimal windows for each factor. The strength of Taguchi methodology lies in the orthogonal array design. Each level of each factor occurs in an equal number of times across the entire array. When compared to factorial design, the possible savings are apparent, the same number of factors and levels examined with factorial 
design would require 256 experiments, whereas in case of Taguchi only 16 experiments are required ${ }^{8}$.

The main objective of this research is the determination of $\mathrm{Cu}$ (II). To optimize the variables simultaneously, Taguchi experiment is used. All factors such as absorbent dosage, $\mathrm{pH}$, time and flow rate are investigated.

\section{EXPERIMENTAL}

The analysis was carried out using an atomic SRECTRAA model 220 (VARIAN), equipped with a flame burner. Operational parameters for metal under study, including lamp currents and wavelength were those recommended by the manufacturer. $\mathrm{Cu}$ (II) was measured under optimized operating conditions by flame atomic absorption spectrometry (FAAS) with an air-acetylene flame. The $\mathrm{pH}$ values in the aqueous phase were measured with METTLER-TOLEDO 225 model $\mathrm{pH}$ meter. The following buffers were used to control the $\mathrm{pH}$ of the solutions: $\mathrm{CH}_{3} \mathrm{COONH}_{4}-\mathrm{CH}_{3} \mathrm{COOH}(\mathrm{pH}=4-6)$, $\mathrm{CH}_{3} \mathrm{COONH}_{4}(\mathrm{pH}=7), \mathrm{NH}_{4} \mathrm{Cl}-\mathrm{NH}_{3}(\mathrm{pH}=8-10)$.

Ultra water was used throughout the work. All chemicals were of analytical reagent grade. All the plastic and glassware were cleaned by soaking in $10 \% \mathrm{HNO}_{3}$ solution and then rinsed with distilled water prior to use. Nitrate salt of $\mathrm{Cu}(\mathrm{II})$ and $\mathrm{HNO}_{3}$ were purchased from Merck. The nanoporous silica gel column was prepared according to previous articles 9 .

Procedure: Standard solution (1000 mg/L) of Cu(II) was prepared. The working standard solutions were prepared by diluting proper volumes of stock standard solution.

$10 \mathrm{~mL}$ of solutions were injected in to column with different of amounts of concentrations and $\mathrm{pH}$. Modified nanoporous eluted by $\mathrm{HNO}_{3}$ and absorbed $\mathrm{Cu}$ (II) was measured by FAAS.

Multivariate experimental design: The central composite design (CCD) was applied to investigate the effects of experimental parameters in preconcentration technique. To achieve optimum parameters, 9 experiments $\left(\mathrm{L}_{9}\right)$ by three onus repeat were performed, at which variables were codified in three levels.

The response surface was built by using Taguchi technique, based on the experimental data obtained for determination $\mathrm{Cu}(\mathrm{II})$ by flame atomic absorption spectrometer.

\section{RESULTS AND DISCUSSION}

It has to be maintained that some properties of parameters are essential in determination of heavy metal ions. However, it is quite difficult to carry out an experimental design including all these factors because of the large number of experiments and complex data analysis required, therefore, the most important factors were chosen. It was found that the four factors i.e., absorption dosage, $\mathrm{pH}$, time and flow rate, have the most significant effect on determination.

Central composite design: According to $\mathrm{CCD}$ approach Table-1 condenses the level for each factor involved in the design strategies. These levels were chosen according to preliminary experiments carried out.

Taguchi method: Using Taguchi technique and considering the data of Table-1, table was drawn (Table-2). According to this Table each run was repeated three times and the

\begin{tabular}{lccc}
\hline \multicolumn{4}{c}{ TABLE-1 } \\
\multicolumn{4}{c}{ RANGE OF THE PARAMETER VARIATION } \\
USED IN THE CENTRAL COMPOSITE DESIGN \\
\hline \multicolumn{1}{c}{ Parameter } & Low level (-1) & Center level $(0)$ & High level $(+1)$ \\
\hline [Absorbent] & 3.0 & 5.0 & 10.0 \\
$\mathrm{pH}$ & 4.0 & 6.0 & 8.0 \\
Time & 1.0 & 2.0 & 5.0 \\
Flow rate & 2.0 & 5.0 & 10.0 \\
\hline
\end{tabular}

TABLE-2

EXPERIMENTAL DATA IN CENTRAL COMPOSITE DESIGN

\begin{tabular}{ccccccc}
\hline Ran & [Absorbent] & Time & $\begin{array}{c}\text { Flow } \\
\text { rate }\end{array}$ & $\mathrm{pH}$ & $\begin{array}{c}\text { Recovery } \\
(\%)\end{array}$ & $\mathrm{S} / \mathrm{N}$ \\
\hline 1 & 3.0 & 1.0 & 2.0 & 4.0 & 18.4 & 25.3 \\
2 & 3.0 & 2.0 & 5.0 & 6.0 & 79.1 & 38.0 \\
3 & 3.0 & 5.0 & 10.0 & 8.0 & 84.2 & 38.5 \\
4 & 5.0 & 2.0 & 10.0 & 4.0 & 24.4 & 27.7 \\
5 & 5.0 & 5.0 & 2.0 & 6.0 & 83.3 & 38.4 \\
6 & 5.0 & 1.0 & 5.0 & 8.0 & 93.1 & 39.4 \\
7 & 10.0 & 5.0 & 5.0 & 4.0 & 24.4 & 27.7 \\
8 & 10.0 & 1.0 & 10.0 & 6.0 & 93.8 & 39.4 \\
9 & 10.0 & 2.0 & 2.0 & 8.0 & 96.3 & 39.7 \\
\hline
\end{tabular}

response surface was obtained. Average effect of each factor on response surface data is shown in Fig. 1. As seen in this figure the average maximum for absorption dosage, $\mathrm{pH}$ and flow rate is at the highest level and time in the lowest level.

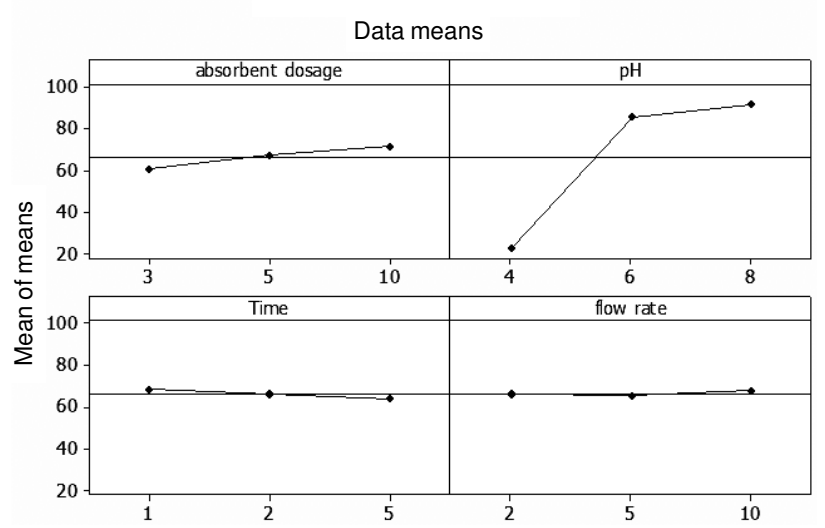

Fig. 1. Effect (a) absorbent dosage, (b) pH, (c) time and (d) flow rate of elutant on response surface

ANOVA analysis: Table-3 shows the amount of sum square and the mean of the square factors. According to these results, it is clear that $\mathrm{pH}$ and absorption dosage have the greatest effects, meanwhile, time and flow rate have the minimum effects on the preconcentration. We also calculated the amounts of variance ratio and the percentage contributed to each factor, which is listed in Table-3. It was found that the F values for all factors are less than $\mathrm{F}$ values derived from statistical values ( $p$ $=0.05, \mathrm{~F}=5.14)$.

TABLE-3

AMOUNT OF SUM SQUARE; MEAN OF SQUARE, VARIENCE RATIO AND PERCENTAGE CONTRIBUTE OF EACH FACTOR

\begin{tabular}{lccccc}
\hline Factor & DOF & SS & Mean Sq & $\mathrm{F}$ & $\mathrm{P}(\%)$ \\
\hline Absorbent & 2 & 180.9 & 90.5 & 0.1 & 2.0 \\
Time & 2 & 8736.1 & 4368.0 & 1.2 & 97.6 \\
pH & 2 & 30.3 & 15.1 & 0.0 & 0.3 \\
Flow rate & 2 & 6.1 & 3.1 & 0.0 & 0.1 \\
\hline
\end{tabular}


So the $\mathrm{H}_{0}$ hypothesis cannot be refused, since this factor is significantly full active. The following Table shows the $p$ values, with considering the ratio of pure SS to total SS share of each factor can be explained. It can be concluded that $\mathrm{pH}$, absorption dosage, time and flow rate have the greatest effects on nano porous efficiency.

$\mathrm{S} / \mathrm{N}$ ratio: We examined the effect of various factors on the $\mathrm{S} / \mathrm{N}$ ratio, which is shown in Fig. 2 and summarized in Table-2. It can be seen in the table related to the maximum $\mathrm{S} / \mathrm{N}$ of 9 tests, which absorption dosage and $\mathrm{pH}$ are in high levels, whereas time is in central and flow rate is at low level. According to Fig. 2 maximum $\mathrm{S} / \mathrm{N}$ is achieved when absorption dosage $=10.0 \mathrm{mg} / \mathrm{L}, \mathrm{pH}=8.0$, time $=2 \mathrm{~min}$, flow rate $=$ $10.0 \mathrm{~mL} / \mathrm{min}$, also the amount of $\mathrm{Cu}(\mathrm{II})$ absorption by nano porous, as a solid phase extraction in these conditions is $96.3 \%$.

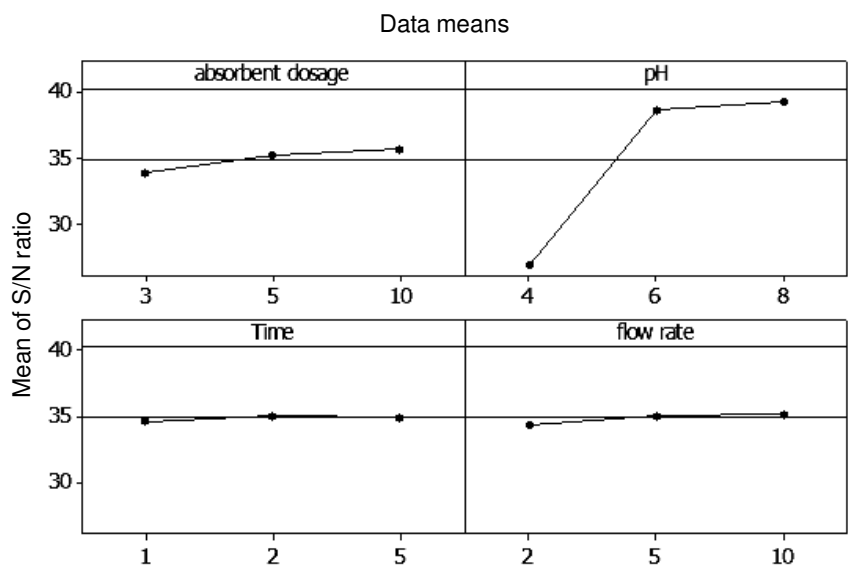

Fig. 2. S/N ratio plot showing effect of (a) absorbent dosage, (b) $\mathrm{pH}$, (c) time and (d) flow rate

\section{Screening of effects}

Effect of sample dosage: In order to investigate the possibility of enrichment of trace quantities of analyt, the effect of sample concentration on the recovery of metal ion was studied by passing 3-10 $\mathrm{mg} / \mathrm{L}$ of sample solution. The maximum recovery was obtained when the concentration of $\mathrm{Cu}$ (II) was equal to $10 \mathrm{mg} / \mathrm{L}$.

Effect of pH: The pH values play an important role for the quantitative recoveries of the metal ions during the solid phase extraction procedure ${ }^{10-16}$. The $\mathrm{Cu}(\mathrm{II})$ solution was adjusted to a desired $\mathrm{pH}$ (between 4-8) and passed through a nanoporous column silica gel. The analyte was then eluted using $10 \mathrm{~mL} \mathrm{HNO}_{3}$ from the column and determined by FAAS, which gave the percentage recoveries of the eluted $\mathrm{Cu}$ (II) at various $\mathrm{pH}$. The retention experiments were carried out in triplicate to determine the precision of the method. According to Fig. 1 the $\mathrm{Cu}(\mathrm{II})$ retention on nanoporous silica gel depends highly on the $\mathrm{pH}$ of the sample solution. The achieved optimum
$\mathrm{pH}$ was equal to 8 . The silica gel is prone to hydrolysis at high $\mathrm{pH}$.

Effect of flow rate: The degree of metal ion absorbed on silica gel was studied at various flow rates $(2-10 \mathrm{~mL} / \mathrm{min})$. The optimum flow rate for $\mathrm{Cu}(\mathrm{II})$ is $10.0 \mathrm{~mL} / \mathrm{min}$. To avoid the long time extraction, the flow rate lower than $2.0 \mathrm{~mL} / \mathrm{min}$ were not employed. However, there was a decrease in the percentage of extraction of $\mathrm{Cu}(\mathrm{II})$ at flow rate higher than $10 \mathrm{~mL} / \mathrm{min}$, because the heavy metal ion cannot be absorbed sufficiently by the silica gel, therefore, the flow rate was maintained at $10 \mathrm{~mL} / \mathrm{min}$ throughout the column ${ }^{17}$.

Effect of time: The effect of time is another important factor in recovery of $\mathrm{Cu}(\mathrm{II})$. The contact time varied from 1-5 $\min$. The amount of optimum time of $2 \mathrm{~min}$ for $\mathrm{Cu}$ (II) was found. Hence, 2 min of contact was enough to reach maximum values of preconcentration.

\section{Conclusion}

A solid phase extraction based on nano porous was used for determination of low level $\mathrm{Cu}$ (II) in aqueous solution by flame atomic absorption. The Taguchi design used in this study has showed to be a valuable tool to achieve optimum conditions. According to ANOVA analysis, time and absorbent dosage parameters have the most effect on separation of $\mathrm{Cu}$ (II). The comparison of the results of applied method, including results of one factor at a time optimization, indicates that Taguchi technique is a good method to predict the behaviour of solid phase extraction.

\section{ACKNOWLEDGEMENTS}

The authors thanks the Research Council at the Islamic Azad University, North Tehran Branch.

\section{REFERENCES}

1. M. Yang, J.X. Li and J.H. Wang, Talanta, 2, 1710 (2007).

2. Y.L. Yu, Z. Du and J.H. Wang, Chin. Anal. Chem., 35, 431 (2007).

3. G. Venkatesh and A.K. Singh, Talanta, 71, 282 (2007).

4. C.F. Poole, Encyclopedia of Separation Science, Academic Press, vol. 3, p. 1405. (2000)

5. V. Camel, Spectrochim. Acta B, 58, 1177 (2008).

6. S.V. Mohan, K. Sirisha, R.S. Rao and P.N. Sarma, Ecotoxicol. Environ. Safety, 68, 252 (2007).

7. J. Antony, Sens. Rev., 26, 227 (2006).

8. K.N. Ballantyne, R.A. Van Oorschot and R.J. Mitchell, Genetics Suppl. Series, 1, 7 (2008).

9. M. Javanbakht, A.R. Badiei, M.R. Ganjali, P. Nourozi, A. Hasheminasab and A. Abdouss, Anal. Chim. Acta, 601, 172 (2007).

10. M. Tuzen, K.O. Saygi, C. Usta and M. Soylak, Bioresour. Technol., 99, 1563 (2008).

11. M. Tuzen, K.O. Saygi and M. Soylak, J. Hazard. Mater, 152, 632 (2008).

12. M. Tuzen and M. Soylak, J. Hazard. Mater., 147, 219 (2007).

13. X. Zhu, M. Wu, J. Sun and X. Zhang, Anal. Lett., 41, 2186 (2008).

14. M. Ghaedi, K. Nikname, A. Shokrollahi, E. Nikname, H. Ghaedi and M. Soylak, J. Hazard. Mater., 158, 131 (2008).

15. P. Liang, Y. Liu and J. Zeng, J. Anal. Atom. Spectrom., 19, 1489 (2004).

16. D. Afzali and A. Mostafavi, Anal. Sci., 24, 1135 (2008).

17. F.Z. Xie, X.C. Lin, X.P. Wu and Z.H. Xie, Talanta, 74, 836 (2008) 